Research

\title{
AURKA gene polymorphisms and central nervous system tumor susceptibility in Chinese children
}

\author{
Yong-Ping Chen ${ }^{1} \cdot$ Li Yuan $^{2} \cdot$ Hui-Ran Lin ${ }^{3} \cdot$ Xiao-Kai Huang ${ }^{4} \cdot$ Ji-Chen Ruan ${ }^{4} \cdot$ Zhen-Jian Zhuo $^{1,5}$
}

Received: 19 August 2021 / Accepted: 17 November 2021

Published online: 15 December 2021

(c) The Author(s) 2021 OPEN

\begin{abstract}
Introduction Central nervous system (CNS) tumors comprise $15-20 \%$ of all malignancies occurring in childhood and adolescence. Previous researches have shown that overexpression and amplification of the AURKA gene could induce multiple human malignancies, with which the connection of CNS tumor susceptibility has not been extensively studied. Material and methods In this study, we assessed whether and to what extent AURKA gene single nucleotide polymorphisms (SNPs) (rs1047972 C > T, rs2273535 T > A, rs8173 G >C) were associated with CNS tumor susceptibility, based on a case-control analysis in 191 CNS tumor patients and 248 controls. We determined this correlation using odds ratios (ORs) and $95 \%$ confidence intervals (Cls).

Results AURKA gene rs $8173 \mathrm{G}>\mathrm{C}$ exhibited a crucial function to CNS tumor susceptibility fall-off (GC/CC vs. GG: adjusted $\mathrm{OR}=0.68,95 \% \mathrm{Cl}=0.46-0.998, P=0.049$ ). In addition, the combined effect of lowering the risk of developing CNS tumors was more pronounced in carriers with 3 protective genotypes than others (adjusted $\mathrm{OR}=0.55,95 \% \mathrm{Cl}=0.31-0.98$, $P=0.044$ ). Further stratification analysis illustrated that the existence of $\mathrm{rs} 8173 \mathrm{GC} / \mathrm{CC}$ and three protective genotypes lowered CNS tumor risk in some subgroups.

Conclusions Our research suggested that the AURKA gene rs $8173 \mathrm{G}>\mathrm{C}$ could significantly reduce CNS tumor susceptibility in Chinese children. More functional experiments are needed to explore the role of the AURKA gene rs8173 G $>C$.
\end{abstract}

Keywords AURKA $\cdot$ Chinese $\cdot$ CNS tumor $\cdot$ Polymorphism $\cdot$ Risk

Yong-Ping Chen and Li Yuan contributed equally to this work

Supplementary Information The online version contains supplementary material available at https://doi.org/10.1007/s12672-021-00459w.

$\triangle$ Ji-Chen Ruan, ruanjichen@163.com; $₫$ Zhen-Jian Zhuo, zhenjianzhuo@163.com | Department of Pediatric Surgery, Guangzhou Institute of Pediatrics, Guangdong Provincial Key Laboratory of Research in Structural Birth Defect Disease, Guangzhou Women and Children's Medical Center, Guangzhou Medical University, 9 Jinsui Road, Guangzhou 510623, Guangdong, China. ${ }^{2}$ Department of Pathology, Guangzhou Women and Children's Medical Center, Guangzhou Medical University, Guangzhou 510623, Guangdong, China. ${ }^{3}$ Faculty of Medicine, Macau University of Science and Technology, Macau 999078, China. ${ }^{4}$ Department of Hematology, The Second Affiliated Hospital and Yuying Children's Hospital of Wenzhou Medical University, 109 West Xueyuan Road, Wenzhou 325027, Zhejiang, China. ${ }^{5}$ Laboratory Animal Center, School of Chemical Biology and Biotechnology, Peking University Shenzhen Graduate School, Shenzhen 518055, Guangdong, China.

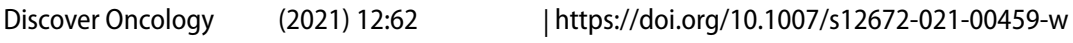




\section{Introduction}

Central nervous system (CNS) tumors represent the most common solid malignancy in childhood [1, 2]. Glioma, thought to root in neural stem cells, glial progenitors, and astrocytes, constituted approximately $50 \%$ of pediatric tumors and $80 \%$ of malignancies among children aged $0-14$ years $[3,4]$. The most common histologic type of gliomas in children and adolescents was pilocytic astrocytoma, which accounted for $29.4 \%[5,6]$. However, in the population as a whole, the highest incidence of gliomas was glioblastoma (3.22 per 100,000 population), representing about $15 \%$ of total primary brain tumors and other CNS tumors, $48.3 \%$ of primary malignant cerebral tumors, and $57.3 \%$ of all gliomas [4].

Most patients with CNS tumors show more than one symptom during the disease. The first most frequent symptom of many patients with high-grade gliomas is a headache. The second most common symptom is vomiting, mainly accompanied by weakness, seizures, memory loss, gait disorder, and behavioral difficulties. Concerning low-grade gliomas in children, they usually present stunting and extreme emaciation [7, 8]. Seriously, CNS tumors can develop complications affecting patients' quality of life like endocrinopathies, infections, venous thromboembolism, and intracerebral edema [9]. As the most representative CNS tumors, gliomas are divided into circumscribed gliomas (WHO grade I) and diffusely infiltrating gliomas (WHO grade II-IV). The former is benign and can be resected completely to achieve healing, while the latter is barely remedied by resection alone, requiring adjuvant therapy such as radio- and chemo-therapy. Additionally, most low-grade gliomas and almost all high-grade glioma are relapsed ultimately, even developing into higher-grade gliomas [10].

CNS tumors can harm patients' health. The damage not only results from cancer itself but the influence of treatment. Patients may suffer functional defects in motor, communication, and neurocognitive skills according to the impact of tumorous location and size. Acute dysfunction is likely to occur after surgical removal. Radiotherapy and chemotherapy are associated with long-term impaired neurocognitive and various toxic reactions, respectively. Moreover, ill effects such as fatigue and nausea can come with drug therapy. Patients with CNS tumors also face a substantial negative impact on their psychology [11].

Antecedently, research reports have discussed that in addition to inherited hazard elements, the other hazard elements of CNS tumors might include several environmental exposures, such as ionizing radiation, allergies, atopic diseases, and mobile phones. It was remarkable that brain-ionizing irradiation was considered a well-defined risk factor for brain tumors [12-14]. Among the genetic risk factors, seven genetic variants have been identified that increased the risk of CNS tumors [15]. TERT, MPHOSPH6, ACYP2, and ZNF208 genes have been identified to impact the prognosis of CNS tumors [16]. However, these genetic mutations are insufficient to clarify the pathogenesis of CNS tumors, and more genetic variants need to be identified.

AURKA (STK15/BTAK), a member of the Aurora families, encodes the mitotic serine/threonine kinase and affects cell-cycle regulation, particularly mitotic process [17]. It is an oncogene located on chromosome 20q13.2 [18]. During the $S$ phase of the cell cycle, AURKA is situated on the centrosomes and takes part in cell division by regulating the formation and separation of mitotic spindles [19]. Therefore, once AURKA is overexpressed, disrupting the assembly of the mitotic checkpoint complex, and forming defective chromatid separation, brings about abnormal mitosis and tumorigenesis [20,21]. Moreover, deficiency of the AURKA gene can lead to damage in cell mitosis, cytokinesis, and genome [22]. Consequently, it is assumed that the AURKA gene can be a potential target for anticancer therapies, and small molecule inhibitors of AURKA attract increasing attention $[19,23]$.

For the past few years, many studies have linked AURKA to human cancers. For example, AURKA has momentous functions in the progression of gastrointestinal cancer risk [24]. Moreover, AURKA, an independent factor, is regarded as a prognostic marker in colorectal adenocarcinoma [26]. Single nucleotide polymorphisms (SNPs) are caused by mutations in a single base, characterized by substituting one nucleotide for another, resulting in polymorphism at the locus with variations [27]. Many previous studies demonstrated that SNPs in mitotic checkpoint genes like AURKA could alter tumorigenesis susceptibility. AURKA gene single nucleotide polymorphisms (SNPs) may be a biomarker for hepatocellular carcinoma [25]. The polymorphism rs 911160 in AURKA was identified to impact gastric cancer [28]. Furthermore, AURKA Ile31 polymorphism was associated with altered transcriptional levels among patients with prostates [29].

Given the importance of these three AURKA gene SNPs to altered cancer risk, and the correlation of these AURKA gene polymorphisms with CNS tumors has not been studied. Therefore, we chose these AURKA SNPs to evaluate for the first time whether these AURKA genetic polymorphisms are associated with CNS tumor risk and the relationship's strength in Chinese children. 


\section{Methods}

\subsection{Sample selection}

The cases and controls were frequency-matched for age, gender and ethnicity. They were enrolled between 2005 and 2019 from Guangzhou Women and Children's Medical Center, the Second Affiliated Hospital, and Yuying Children's Hospital of Wenzhou Medical University. Every patient in the case group was confirmed with CNS tumors. The inclusion criteria for CNS tumors patients were biopsy verified or histologically confirmed glioma. The biopsy requires ample tumor tissue derived from the contrast-enhancement margin of the lesion instead of necrotic core and subsequently histological diagnose [30]. Histologically classification is served as the gold standard to determine CNS tumors. Briefly, CNS tumors are diagnosed histologically by WHO classification. Grade I CNS tumors grow slowly and have well-demarcated tumor cells. Grade II CNS tumors also develop slowly but usually exhibit brain-invasive growth, which cannot be resected entirely. Grade III CNS tumors are characterized by anaplasia histologically, showing rapid growth, particularly high cellularity, cellular pleomorphism, and elevated mitotic activity. Grade IV CNS tumors are glioblastomas, microscopically different from grade III CNS tumors, which display microvascular proliferation and necrosis [11]. The control group with no history of malignancy was derived from those undergoing conventional check-up during the same period. The frequency distribution of chosen variables between the case and controls was listed in Additional file 1: Table S1. The parents or guardians of all subjects offered valid informed consent before this research. Furthermore, we conducted this investigation under the approval of the Institutional Review Board of the Guangzhou Women and Children's Medical Center (No. 2016021650) in Feb 2016 and The Second Affiliated Hospital and Yuying Children's Hospital of Wenzhou Medical University (No. LCKY2019-165) in May 2019.

\subsection{Polymorphism selection and genotyping}

We selected single nucleotide polymorphisms based on the established criteria in the previous studies [31, 32]. In general, the underlying SNPs were chosen in the $5^{\prime}$-flanking region, $3^{\prime}$ - and $5^{\prime}$ - untranslated regions, and the exons of the AURKA gene. Finally, we decided rs 1047972 C > T, rs2273535 T > A, and rs8173 G > C in the AURKA gene to investigate their association with CNS tumor susceptibility in Chinese children. Using the TIANamp Blood DNA Kit (TianGen Biotech, Beijing, China), genomic DNA was isolated from the peripheral blood specimens of participants. As mentioned before $[31,32]$, the TaqMan real-time PCR was utilized in genotyping through a $7900 \mathrm{HT}$ sequence detection system (Applied Biosystem, Foster City, CA).

\subsection{Statistical analysis}

The differences between genotypes frequencies in the controls and Hardy-Weinberg equilibrium (HWE) were measured via goodness-of-fit $\chi^{2}$ test. The two-sided $\chi^{2}$ test was conducted to compare the discrepancies in the distributions of participants' selected variables. Odds ratios (ORs) and 95\% confidence intervals (Cls) were calculated using logistic regression analysis. Association of AURKA gene polymorphisms with CNS tumor risk was evaluated using ORs and $95 \%$ $\mathrm{Cls}$ adjusted for age and gender. $P$-value was less than 0.05 , implying statistically significant. We performed the combined effect of genotypes using logistic regression analysis, subsequently evaluated the impact on CNS tumor risk by agegender adjusted OR, $95 \% \mathrm{Cl}$, and $P$-value. The statistical analysis was staged on SAS v10.0 (SAS Institute, Cary, NC, USA).

\section{Results}

\subsection{Effect of AURKA gene SNPs on CNS tumor risk}

The chosen AURKA SNPs in our research are all located on chromosome 20. The rs 1047972 is localized in exon 3:56, 386, 407, in which the nucleotide substitution usually exists between T and C. The rs 2273535 is localized in exon 3: 56, 386, and its nucleotide substitution is $A / C, A / G$, and $A / T$. The rs 8173 is localized in exon 9:56, 369, 735, and the nucleotide substitution is C/G and C/T. 191 cases and 248 controls were successfully genotyped to investigate the impact of rs 1047972, rs2273535, and rs8173 on CNS tumor risk in this study. All genotype frequency distributions of these SNPs conformed 
to Hardy-Weinberg equilibrium (HWE $=0.567$ for rs 1047972; HWE $=0.328$ for rs2273535; HWE $=0.332$ for rs8173). Table 1 included detailed results of these three SNPs' genotype frequencies and their association with CNS tumor susceptibility. Of note, rs8173 G > C exhibited a prominently correlation with decreased CNS tumor risk under the dominant model (GC/CC vs. GG: adjusted $\mathrm{OR}=0.68,95 \% \mathrm{Cl}=0.46-0.998, P=0.049$ ), which means the effect of $\mathrm{GC} / \mathrm{CC}$ genotypes was more protective than $\mathrm{GG}$ genotype after the $C$ allele was regarded as mutant type. No significant associations with CNS tumor risk were observed in the rest of SNPs ( $r$ s $1047972 \mathrm{C}>\mathrm{T}$, rs $2273535 \mathrm{~T}>\mathrm{A}$ ). The combined effect of protective genotypes showed that participants with 3 protective genotypes had a 0.55 -fold (adjusted $\mathrm{OR}=0.55,95 \% \mathrm{Cl}=0.31-0.98, P=0.044$ ) reduction in the development of CNS tumors. Subjects with 1, 2 protective genotypes could not impact CNS tumor risk. A similar negative result was detected for 3 protective genotypes in comparison to $0-2$ protective genotypes.

\subsection{Stratification analysis}

Our stratified analysis discovered the relationship between rs $8173 \mathrm{G}>\mathrm{C}$ polymorphism and CNS tumor susceptibility based on age, gender, subtypes, and clinical stage (Table 2). In comparison to GG genotype carriers, GC/CC genotype carriers were less susceptible to CNS tumors in males (adjusted $\mathrm{OR}=0.47,95 \% \mathrm{Cl}=0.28-0.80, P=0.005$ ) and clinical

Table 1 Association between AURKA gene polymorphisms and CNS tumor susceptibility in Chinese children

\begin{tabular}{|c|c|c|c|c|c|c|c|}
\hline Genotype & $\begin{array}{l}\text { Cases } \\
(\mathrm{N}=191)\end{array}$ & $\begin{array}{l}\text { Controls } \\
(\mathrm{N}=248)\end{array}$ & $P^{a}$ & $\begin{array}{l}\text { Crude OR } \\
(95 \% \mathrm{Cl})\end{array}$ & $P$ & $\begin{array}{l}\text { Adjusted OR } \\
(95 \% \mathrm{Cl})^{\mathrm{b}}\end{array}$ & $P^{\mathrm{b}}$ \\
\hline \multicolumn{8}{|c|}{ rs $1047972 \mathrm{C}>\mathrm{T}(\mathrm{HWE}=0.567)$} \\
\hline $\mathrm{CC}$ & $157(82.20)$ & $190(76.61)$ & & 1.00 & & 1.00 & \\
\hline $\mathrm{CT}$ & $30(15.71)$ & $53(21.37)$ & & $0.69(0.42-1.12)$ & 0.134 & $0.67(0.41-1.10)$ & 0.115 \\
\hline TT & $4(2.09)$ & $5(2.02)$ & & $0.97(0.26-3.67)$ & 0.962 & $0.93(0.24-3.54)$ & 0.910 \\
\hline Additive & & & 0.221 & $0.77(0.51-1.17)$ & 0.222 & $0.76(0.50-1.15)$ & 0.188 \\
\hline Dominant & $34(17.80)$ & $58(23.39)$ & 0.154 & $0.71(0.44-1.14)$ & 0.155 & $0.69(0.43-1.12)$ & 0.130 \\
\hline Recessive & $187(97.91)$ & $243(97.98)$ & 0.954 & $1.04(0.28-3.93)$ & 0.954 & $1.00(0.26-3.80)$ & 0.997 \\
\hline \multicolumn{8}{|c|}{$\mathrm{rs} 2273535 \mathrm{~T}>\mathrm{A}(\mathrm{HWE}=0.328)$} \\
\hline TT & $88(46.07)$ & $105(42.34)$ & & 1.00 & & 1.00 & \\
\hline $\mathrm{TA}$ & $87(45.55)$ & $118(47.58)$ & & $0.88(0.59-1.31)$ & 0.526 & $0.90(0.60-1.34)$ & 0.589 \\
\hline AA & $16(8.38)$ & $25(10.08)$ & & $0.76(0.38-1.52)$ & 0.443 & $0.76(0.38-1.53)$ & 0.444 \\
\hline Additive & & & 0.380 & $0.88(0.65-1.18)$ & 0.380 & $0.88(0.66-1.19)$ & 0.408 \\
\hline Dominant & $103(53.93)$ & $143(57.66)$ & 0.435 & $0.86(0.59-1.26)$ & 0.435 & $0.87(0.60-1.28)$ & 0.484 \\
\hline Recessive & $175(91.62)$ & $223(89.92)$ & 0.543 & $0.82(0.42-1.58)$ & 0.544 & $0.81(0.42-1.57)$ & 0.525 \\
\hline \multicolumn{8}{|c|}{ rs8173 G >C (HWE = 0.332) } \\
\hline GG & $85(44.50)$ & $87(35.08)$ & & 1.00 & & 1.00 & \\
\hline GC & $86(45.03)$ & $124(51.21)$ & & $0.69(0.46-1.04)$ & 0.076 & $0.70(0.46-1.05)$ & 0.082 \\
\hline CC & $20(10.47)$ & $34(13.71)$ & & $0.60(0.32-1.13)$ & 0.113 & $0.60(0.32-1.13)$ & 0.116 \\
\hline Additive & & & 0.048 & $0.75(0.56-0.998)$ & 0.049 & $0.75(0.56-1.00)$ & 0.051 \\
\hline Dominant & $106(55.50)$ & $161(64.92)$ & 0.045 & $0.67(0.46-0.99)$ & 0.045 & $0.68(0.46-0.998)$ & 0.049 \\
\hline Recessive & $171(89.53)$ & $214(86.29)$ & 0.306 & $0.74(0.41-1.33)$ & 0.307 & $0.73(0.41-1.33)$ & 0.306 \\
\hline \multicolumn{8}{|c|}{ Combined effect of protective genotypes ${ }^{c}$} \\
\hline 0 & $69(36.13)$ & $74(29.84)$ & 0.080 & 1.00 & & 1.00 & \\
\hline 1 & $27(14.14)$ & $36(14.52)$ & & $0.80(0.44-1.46)$ & 0.475 & $0.78(0.43-1.43)$ & 0.424 \\
\hline 2 & $69(36.13)$ & $88(35.48)$ & & $0.84(0.53-1.33)$ & 0.455 & $0.85(0.54-1.35)$ & 0.490 \\
\hline 3 & $26(13.61)$ & $50(20.16)$ & & $0.56(0.31-0.99)$ & 0.047 & $0.55(0.31-0.98)$ & 0.044 \\
\hline $0-2$ & $165(86.39)$ & $198(79.84)$ & & 1.00 & & 1.00 & \\
\hline 3 & $26(13.61)$ & $50(20.16)$ & 0.072 & $0.62(0.37-1.05)$ & 0.074 & $0.62(0.37-1.04)$ & 0.068 \\
\hline
\end{tabular}

Bold indicates significant results with $P<0.05$

$O R$ odds ratio, $\mathrm{Cl}$ confidence interval, HWE Hardy-Weinberg equilibrium

${ }^{\mathrm{a}} \chi^{2}$ test for genotype distributions between glioma patients and cancer-free controls

${ }^{\mathrm{b}}$ Adjusted for age and gender

'Protective genotypes were carriers with rs 1047972 CT/TT, rs2273535 TA/AA, rs8173 GC/CC genotypes 
Table 2 Stratification analysis of risk genotypes with CNS tumor susceptibility

\begin{tabular}{|c|c|c|c|c|c|c|c|c|c|}
\hline \multirow[t]{3}{*}{ Variables } & \multirow{2}{*}{\multicolumn{2}{|c|}{$\begin{array}{l}\text { rs8173 } \\
\text { (cases/controls) }\end{array}$}} & \multirow[t]{3}{*}{$\operatorname{AOR}(95 \% \mathrm{CI})^{\mathrm{a}}$} & \multirow[t]{3}{*}{$P^{\mathrm{a}}$} & & \multirow{2}{*}{\multicolumn{2}{|c|}{$\begin{array}{l}\text { Protective geno- } \\
\text { types } \\
\text { (cases/controls) }\end{array}$}} & \multirow[t]{3}{*}{$\operatorname{AOR}(95 \% \mathrm{Cl})^{\mathrm{a}}$} & \multirow[t]{3}{*}{$P^{a}$} \\
\hline & & & & & & & & & \\
\hline & GG & $\mathrm{GC} / \mathrm{CC}$ & & & & $0-2$ & 3 & & \\
\hline \multicolumn{10}{|l|}{ Age, month } \\
\hline$<60$ & $41 / 44$ & $56 / 82$ & $0.73(0.42-1.26)$ & 0.261 & & $84 / 99$ & $13 / 27$ & $0.57(0.27-1.17)$ & 0.123 \\
\hline$\geq 60$ & $44 / 43$ & $50 / 79$ & $0.61(0.35-1.06)$ & 0.080 & & $81 / 99$ & $13 / 23$ & $0.68(0.33-1.44)$ & 0.317 \\
\hline \multicolumn{10}{|l|}{ Gender } \\
\hline Females & $33 / 40$ & $56 / 64$ & $1.06(0.59-1.92)$ & & 0.837 & $74 / 84$ & $15 / 20$ & $0.82(0.39-1.73)$ & 0.606 \\
\hline Males & $52 / 47$ & $50 / 97$ & $0.47(0.28-0.80)$ & & 0.005 & $91 / 114$ & $11 / 30$ & $0.47(0.22-0.99)$ & 0.046 \\
\hline \multicolumn{10}{|l|}{ Subtypes } \\
\hline Astrocytic tumors & $61 / 87$ & $75 / 161$ & $0.67(0.43-1.03)$ & & 0.065 & $117 / 198$ & $19 / 50$ & $0.61(0.34-1.09)$ & 0.097 \\
\hline Ependymoma & $14 / 87$ & $19 / 161$ & $0.70(0.33-1.47)$ & & 0.345 & 29/198 & $4 / 50$ & $0.55(0.18-1.64)$ & 0.282 \\
\hline Neuronal and mixed & $6 / 87$ & $8 / 161$ & $0.67(0.22-2.03)$ & & 0.483 & $12 / 198$ & $2 / 50$ & $0.66(0.14-3.08)$ & 0.598 \\
\hline Embryonal tumors & $4 / 87$ & $3 / 161$ & $0.42(0.08-2.16)$ & & 0.300 & $6 / 198$ & $1 / 50$ & $0.64(0.06-6.40)$ & 0.703 \\
\hline \multicolumn{10}{|l|}{ Clinical stages } \\
\hline 1 & $48 / 87$ & $62 / 161$ & $0.69(0.43-1.09)$ & & 0.109 & $93 / 198$ & $17 / 50$ & $0.70(0.38-1.29)$ & 0.258 \\
\hline II & $19 / 87$ & $19 / 161$ & $0.54(0.27-1.07)$ & & 0.079 & $34 / 198$ & $4 / 50$ & $0.46(0.16-1.36)$ & 0.162 \\
\hline III & $6 / 87$ & $11 / 161$ & $0.95(0.34-2.68)$ & & 0.921 & $15 / 198$ & $2 / 50$ & $0.54(0.12-2.45)$ & 0.424 \\
\hline IV & $12 / 87$ & $13 / 161$ & $0.66(0.28-1.58)$ & & 0.354 & $22 / 198$ & $3 / 50$ & $0.50(0.13-1.84)$ & 0.294 \\
\hline$I+I I$ & $67 / 87$ & $81 / 161$ & $0.65(0.43-0.99)$ & & 0.044 & $127 / 198$ & $21 / 50$ & $0.65(0.37-1.13)$ & 0.128 \\
\hline III+IV & $18 / 87$ & $24 / 161$ & $0.77(0.39-1.51)$ & & 0.450 & $37 / 198$ & $5 / 50$ & $0.52(0.19-1.41)$ & 0.201 \\
\hline
\end{tabular}

Bold indicates significant results with $P<0.05$

$A O R$ adjusted odds ratio, $\mathrm{Cl}$ confidence interval

${ }^{a}$ Adjusted for age and gender, omitting the corresponding stratify factor

stages I+ II tumors (adjusted $\mathrm{OR}=0.65,95 \% \mathrm{Cl}=0.43-0.99, P=0.044$ ). Meanwhile, in contrast to subjects with 0 to 2 protective genotypes, those with 3 protective genotypes had a 0.47 -fold reduced risk of developing CNS tumors in males (adjusted $\mathrm{OR}=0.47,95 \% \mathrm{Cl}=0.22-0.99, P=0.046$ ). However, there was no evidence of correlation with CNS tumors in age and tumor subtypes.

\section{Discussion}

This case-control study was performed to analyze the relationship between AURKA gene SNPs and CNS tumor risk in 191 patients and 248 cancer-free controls.

Vast documents have indicated that AURKA ensured normal mitosis by maintaining centrosomes and spindle poles' stability. If the AURKA gene was abnormally expressed, the process was defective, promoting the transformation and progression of several human malignancies. Thus, amplification and overexpression of AURKA were strongly associated with several tumor risks [33-35], particularly evident in certain gene polymorphisms. For example, Wang et al. indicated that genetic variations in AURKA played a predictive role in the early clinical status of hepatocellular carcinoma (HCC). The HCC development was more affected by AURKA gene rs1047972 (V57I) polymorphism (C >T) than other SNPs. The rs 1047972 variant TT genotypes conferred higher HCC risk than CC genotypes [25]. Gastric cancer (GC) was influenced by AURKA rs 1047972. The rs1047972 TT and CC + CT genotypes contributed to elevated GC development risk, especially intestinal, diffuse, and both types of GC, reported by Mesic et al. in 2016 [36]. The AURKA gene rs2273535 (T91A) comes from nucleotide position 91, boosting the transformation of I1e to Phe. The research by Lee et al. revealed that the AURKA rs2273535 variant was susceptible to oral cancer. Additionally, if this SNP were exposed to alcohol, betel quid, and cigarettes, it would combine with them, known as gene-environmental interaction, which could produce synergistic effects to increase oral cancer risk [37]. Furthermore, the correlation between AURKA overexpression and GC poor prognosis was identified by Zhou et al. The AURKA rs2273535 TT genotypes increased higher risk of GC tumorigenesis than AA 
genotypes [38]. These two polymorphisms ( $r$ 1047972 G > A, rs2273535 T > A) were detected in exon 3 of the AURKA gene. It has been studied that rs 1047972 variants modified rs 2273535 polymorphism function and protein secondary structure to reduce carcinogenesis risk. Of note, the protective effect of rs 1047972 was obvious in Caucasians and breast cancer, while not among Asians [39]. In terms of literature on the AURKA gene rs 8173 is rather lacking. Our previous research has reported that rs $8173 \mathrm{G}>C$ was conducive to decreasing children's Wilms tumor susceptibility, especially individuals with rs8173 GC/CC genotypes in subgroups of $>18$ months, male, and clinical stages III + IV [40]. Regrettably, the AURKA polymorphisms were not associated with neuroblastoma risk by another previous study [41]. Other AURKA SNPs such as rs 2064863 and rs6024836 have been found to be related to cancer susceptibility [42, 43].

Thus, although the investigations of how these three AURKA SNPs impact tumor risk are still preliminary, the probability that these SNPs affect CNS tumors cannot be excluded. Given that AURKA SNPs are frequently observed in multiple human malignancies and the implication of AURKA SNPs in CNS tumors has not been investigated intensively. Therefore, we are the first to speculate that the susceptibility of CNS tumors may be affected by AURKA gene SNPs. Existing research demonstrated that Gli2/miR-124/AURKA axis might be the key to influencing AURKA's effect on glioma. Gli2 is a glioma-associated oncogene transcription factor. The target gene of Gli2 can mediate the Hedgehog signaling pathway to facilitate the occurrence of glioma [44]. Briefly, miR-124 is suppressed because the Gli2 combines with the upstream region of miR-124 transcriptional initiation locus excessively, whereas miR-124 existence is relevant to AURKA expression, giving rise to the anomaly of AURKA expression in glioma cells, which ultimately promotes cell proliferation and tumorigenesis $[45,46]$. The Gli2/miR-124/AURKA occupies an essential position during the growth and development of human glioma cells.

In this study, we have shed light on that AURKA rs8173 was associated with reduced CNS tumor risk. At the same time, there were no apparent correlations between other AURKA gene SNPs (rs1047972 and rs2273535) and CNS tumor susceptibility. Moreover, the combined effect of protective genotypes and stratification analysis suggested that only subjects with 3 protective genotypes reduced CNS tumor risk significantly. Besides, stratified analysis manifested carriers with AURKA gene rs $8173 \mathrm{GC} / C C$ were linked to decreased CNS tumor risk in the subgroup of male and clinical stages I+ II diseases, with no findings in age and subtypes. In our study, although, AURKA rs 1047972 revealed negative results for altering CNS tumor risk, AURKA rs 1047972 variants were identified to change lung adenocarcinoma (LADC) development probably [47]. Moreover, the rs 1047972 variants were associated with elevated gastric cancer (GC) risk and basal-like breast cancer $[48,49]$. With no association of AURKA rs 2273535 with CNS tumor susceptibility, AURKA rs2273535 polymorphisms were associated with overall enhanced cancer susceptibility, particularly breast cancer [50]. Furthermore, the SNP was a favorable prognostic factor for urothelial carcinoma patients treated with alisertib (an AURKA inhibitor) [51]. In a previous study, the correlation between AURKA rs 8173 and breast cancer risk was reported by Hong Shi et al. [52]. In addition, the $\mathrm{rs} 8173 \mathrm{G}>\mathrm{C}$ was associated with reduced Wilms tumor risk [40]. This study also indicated the critical role of $A U R K A$ rs 8173 in cancer modification.

However, serval deficiencies in our study deserve to point out. In the first place, what we analyzed was not a large sample size but a relatively small sample size, only containing 191 patients and 248 controls, which was insufficient to produce a convincing statistic. For the sake of reducing fortuitous events to the utmost, more samples are needed for verification. In the second place, we only tested three AURKA SNPs in the research. More potentially functional CNS tumor risk-associated SNPs in the AURKA gene and other genes should be investigated later. Of note, our previous study indicated that FTO gene SNPs are unlikely to have significant effects on CNS tumor risk [53]. In that study, we also included the samples of Guangzhou and Wenzhou. More SNPs in diverse genes are needed to genotype. Finally, the case-control study conducted in this study might have been biased because the genotype distribution of the general population might not be represented by the genotype distribution studied in the hospital.

In this research, AURKA gene rs $8173 \mathrm{G}>C$ polymorphism was identified to confer decreased CNS tumor susceptibility significantly in Chinese children. Continuous studies with a larger sample size are warranted to elucidate the effect of AURKA gene SNPs on CNS tumor risk.

Acknowledgements This study was supported by grants from the National Natural Science Foundation of China (No: 81802346) and China Postdoctoral Science Foundation (No. 2020T130132).

Authors' contributions YPC and LY: concept, design, definition of intellectual content, literature search, experimental studies, data acquisition, data analysis, statistical analysis, manuscript preparation, and manuscript review. XKH: concept, design, literature search, data acquisition, data analysis, statistical analysis, manuscript editing, and manuscript review. JCR and ZJZ take responsibility for the integrity of the work as a whole from inception to published article. All the authors approved the final version of the manuscript. 
Funding This work was funded by National Natural Science Foundation of China (Grant No.: 81802346), China Postdoctoral Science Foundation (Grant No.: 2020T130132).

Data availability All data generated or analyzed during this study are included in this published article and its supplementary information files.

\section{Declarations}

Ethics approval This study was approved by Ethics Committee of Guangzhou Women and Children's Medical Center.

Competing interests No financial or nonfinancial benefits have been received or will be received from any party related directly or indirectly to the subject of this article.

Open Access This article is licensed under a Creative Commons Attribution 4.0 International License, which permits use, sharing, adaptation, distribution and reproduction in any medium or format, as long as you give appropriate credit to the original author(s) and the source, provide a link to the Creative Commons licence, and indicate if changes were made. The images or other third party material in this article are included in the article's Creative Commons licence, unless indicated otherwise in a credit line to the material. If material is not included in the article's Creative Commons licence and your intended use is not permitted by statutory regulation or exceeds the permitted use, you will need to obtain permission directly from the copyright holder. To view a copy of this licence, visit http://creativecommons.org/licenses/by/4.0/.

\section{References}

1. Gusyatiner O, Hegi ME. Glioma epigenetics: from subclassification to novel treatment options. Semin Cancer Biol. 2018;51:50-8. https:// doi.org/10.1016/j.semcancer.2017.11.010.

2. Ostrom QT, et al. The epidemiology of glioma in adults: a "state of the science" review. Neuro Oncol. 2014;16(7):896-913. https://doi.org/ 10.1093/neuonc/nou087.

3. Zong H, Verhaak RG, Canoll P. The cellular origin for malignant glioma and prospects for clinical advancements. Expert Rev Mol Diagn. 2012;12(4):383-94. https://doi.org/10.1586/erm.12.30.

4. Ostrom QT, et al. CBTRUS statistical report: primary brain and other central nervous system tumors diagnosed in the United States in 2014-2018. Neuro Oncol. 2021;23(12 Suppl 2):iii1-105. https://doi.org/10.1093/neuonc/noab200.

5. Dang M, Phillips PC. Pediatric Brain Tumors. Continuum (Minneap Minn). 2017;23(6, Neuro-oncology):1727-57. https://doi.org/10.1212/ con.0000000000000545.

6. Perry A, Wesseling P. Histologic classification of gliomas. Handb Clin Neurol. 2016;134:71-95. https://doi.org/10.1016/b978-0-12-802997-8. 00005-0.

7. Ostrom QT, et al. CBTRUS statistical report: primary brain and other central nervous system tumors diagnosed in the United States in 2012-2016. Neuro Oncol. 2019;21(Suppl 5):v1-100. https://doi.org/10.1093/neuonc/noz150.

8. Udaka YT, Packer RJ. Pediatric brain tumors. Neurol Clin. 2018;36(3):533-56. https://doi.org/10.1016/j.ncl.2018.04.009.

9. Nabors LB, et al. Central nervous system cancers, version 3.2020, NCCN clinical practice guidelines in oncology. J Natl Compr Canc Netw. 2020;18(11):1537-70. https://doi.org/10.6004/jnccn.2020.0052.

10. Lapointe S, Perry A, Butowski NA. Primary brain tumours in adults. Lancet. 2018;392(10145):432-46. https://doi.org/10.1016/s01406736(18)30990-5.

11. Weller M, et al. Glioma. Nat Rev Dis Primers. 2015;1:15017. https://doi.org/10.1038/nrdp.2015.17.

12. Vienne-Jumeau A, Tafani C, Ricard D. Environmental risk factors of primary brain tumors: a review. Rev Neurol (Paris). 2019;175(10):664-78. https://doi.org/10.1016/j.neurol.2019.08.004.

13. Omuro A, DeAngelis LM. Glioblastoma and other malignant gliomas: a clinical review. JAMA. 2013;310(17):1842-50. https://doi.org/10. 1001/jama.2013.280319.

14. Yang $M$, et al. Mobile phone use and glioma risk: a systematic review and meta-analysis. PLoS ONE. 2017;12(5): e0175136. https://doi. org/10.1371/journal.pone.0175136.

15. Ostrom QT, et al. Epidemiology of intracranial gliomas. Prog Neurol Surg. 2018;30:1-11. https://doi.org/10.1159/000464374.

16. Cui Y, et al. The effects of gene polymorphisms on glioma prognosis. J Gene Med. 2017;19(11):345-52. https://doi.org/10.1002/jgm.2989.

17. Li M, et al. The role of Aurora-A in cancer stem cells. Int J Biochem Cell Biol. 2018;98:89-92. https://doi.org/10.1016/j.biocel.2018.03.007.

18. Andrews PD, et al. Mitotic mechanics: the auroras come into view. Curr Opin Cell Biol. 2003;15(6):672-83. https://doi.org/10.1016/j.ceb. 2003.10.013.

19. Yan M, et al. Aurora-A kinase: a potent oncogene and target for cancer therapy. Med Res Rev. 2016;36(6):1036-79. https://doi.org/10. 1002/med.21399.

20. Tong $\mathrm{T}$, et al. Overexpression of Aurora-A contributes to malignant development of human esophageal squamous cell carcinoma. Clin Cancer Res. 2004;10(21):7304-10. https://doi.org/10.1158/1078-0432.Ccr-04-0806.

21. Niu H, et al. Aurora A functional single nucleotide polymorphism (SNP) correlates with clinical outcome in patients with advanced solid tumors treated with alisertib, an investigational Aurora A kinase inhibitor. EBioMedicine. 2017;25:50-7. https://doi.org/10.1016/j.ebiom. 2017.10.015.

22. Kufer TA, Nigg EA, Silljé HH. Regulation of Aurora-A kinase on the mitotic spindle. Chromosoma. 2003;112(4):159-63. https://doi.org/10. 1007/s00412-003-0265-1.

23. Yen CC, et al. Expression profile-driven discovery of AURKA as a treatment target for liposarcoma. Int J Oncol. 2019;55(4):938-48. https:// doi.org/10.3892/ijo.2019.4861. 
24. Katsha A, et al. Aurora kinase $A$ in gastrointestinal cancers: time to target. Mol Cancer. 2015;14:106. https://doi.org/10.1186/ s12943-015-0375-4.

25. Wang B, et al. Variations in the AURKA gene: biomarkers for the development and progression of hepatocellular carcinoma. Int J Med Sci. 2018;15(2):170-5. https://doi.org/10.7150/ijms.22513.

26. Koh HM, et al. Aurora kinase A is a prognostic marker in colorectal adenocarcinoma. J Pathol Transl Med. 2017;51(1):32-9. https://doi. org/10.4132/jptm.2016.10.17.

27. Schork NJ, Fallin D, Lanchbury JS. Single nucleotide polymorphisms and the future of genetic epidemiology. Clin Genet. 2000;58(4):250-64. https://doi.org/10.1034/j.1399-0004.2000.580402.x.

28. Mesic A, et al. Single nucleotide polymorphisms rs911160 in AURKA and rs2289590 in AURKB mitotic checkpoint genes contribute to gastric cancer susceptibility. Environ Mol Mutagen. 2017;58(9):701-11. https://doi.org/10.1002/em.22129.

29. Matarasso N, et al. Functional analysis of the Aurora Kinase A lle31 allelic variant in human prostate. Neoplasia. 2007;9(9):707-15. https:// doi.org/10.1593/neo.07322.

30. Reni M, et al. Central nervous system gliomas. Crit Rev Oncol Hematol. 2017;113:213-34. https://doi.org/10.1016/j.critrevonc.2017.03. 021.

31. Zhuo Z, et al. Correlation between the genetic variants of base excision repair (BER) pathway genes and neuroblastoma susceptibility in eastern Chinese children. Cancer Commun (Lond). 2020. https://doi.org/10.1002/cac2.12088.

32. Zhuo ZJ, et al. Functional polymorphisms at ERCC1/XPF genes confer neuroblastoma risk in Chinese children. EBioMedicine. 2018;30:1139. https://doi.org/10.1016/j.ebiom.2018.03.003.

33. Guo M, et al. Increased AURKA promotes cell proliferation and predicts poor prognosis in bladder cancer. BMC Syst Biol. 2018;12(Suppl 7):118. https://doi.org/10.1186/s12918-018-0634-2.

34. Zhu X, Mei J, Wang Z. Aurora-A kinase: potential tumor marker of osteosarcoma. J Cancer Res Ther. 2014;10(Suppl):C102-7. https://doi. org/10.4103/0973-1482.145804.

35. Nigg EA. Mitotic kinases as regulators of cell division and its checkpoints. Nat Rev Mol Cell Biol. 2001;2(1):21-32. https://doi.org/10.1038/ 35048096.

36. Mesic A, et al. Association of the AURKA and AURKC gene polymorphisms with an increased risk of gastric cancer. IUBMB Life. 2016;68(8):634-44. https://doi.org/10.1002/iub.1521.

37. Lee $\mathrm{CP}$, et al. AURKA Phe31lle polymorphism interacted with use of alcohol, betel quid, and cigarettes at multiplicative risk of oral cancer occurrence. Clin Oral Investig. 2015;19(8):1825-32. https://doi.org/10.1007/s00784-015-1432-5.

38. Zhou X, Wang P, Zhao H. The association between AURKA gene rs 2273535 polymorphism and gastric cancer risk in a Chinese population. Front Physiol. 2018;9:1124. https://doi.org/10.3389/fphys.2018.01124.

39. Tang W, et al. Aurora-A V57I (rs1047972) polymorphism and cancer susceptibility: a meta-analysis involving 27,269 subjects. PLoS ONE. 2014;9(3): e90328. https://doi.org/10.1371/journal.pone.0090328.

40. Lu T, et al. AURKA rs8173 G>C polymorphism decreases Wilms tumor risk in Chinese children. J Oncol. 2019;2019:9074908. https://doi. org/10.1155/2019/9074908.

41. Tang J, et al. Lack of associations between AURKA gene polymorphisms and neuroblastoma susceptibility in Chinese children. 2018. Biosci Rep. https://doi.org/10.1042/bsr20180292.

42. Chou $\mathrm{CH}$, et al. Combined effect of genetic polymorphisms of AURKA and environmental factors on oral cancer development in Taiwan. PLoS ONE. 2017;12(2): e0171583. https://doi.org/10.1371/journal.pone.0171583.

43. Huang $\mathrm{CH}$, et al. Impacts of AURKA genetic polymorphism on urothelial cell carcinoma development. J Cancer. 2019;10(6):1370-4. https:// doi.org/10.7150/jca.30014.

44. Huang D, et al. GLI2 promotes cell proliferation and migration through transcriptional activation of ARHGEF16 in human glioma cells. J Exp Clin Cancer Res. 2018;37(1):247. https://doi.org/10.1186/s13046-018-0917-x.

45. Xu L, et al. Inhibition of the Hedgehog signaling pathway suppresses cell proliferation by regulating the Gli2/miR-124/AURKA axis in human glioma cells. Int J Oncol. 2017;50(5):1868-78. https://doi.org/10.3892/ijo.2017.3946.

46. Qiao W, et al. miR-124 suppresses glioblastoma growth and potentiates chemosensitivity by inhibiting AURKA. Biochem Biophys Res Commun. 2017; 486(1):43-8. https://doi.org/10.1016/j.bbrc.2017.02.120.

47. Yang PJ, et al. Impact of Aurora kinase A polymorphism and epithelial growth factor receptor mutations on the clinicopathological characteristics of lung adenocarcinoma. Int J Environ Res Public Health. 2020. https://doi.org/10.3390/ijerph17197350.

48. Mesic A, et al. Characterization and risk association of polymorphisms in Aurora kinases $A$, B and $C$ with genetic susceptibility to gastric cancer development. BMC Cancer. 2019;19(1):919. https://doi.org/10.1186/s12885-019-6133-z.

49. Taylor NJ, et al. Genetic variation in cell cycle regulatory gene AURKA and association with intrinsic breast cancer subtype. Mol Carcinog. 2015;54(12):1668-77. https://doi.org/10.1002/mc.22238.

50. Wang S, et al. AURKA rs $2273535 \mathrm{~T}>$ A polymorphism associated with cancer risk: a systematic review with meta-analysis. Front Oncol. 2020;10:1040. https://doi.org/10.3389/fonc.2020.01040.

51. Necchi A, et al. Association of an aurora kinase a (AURKA) gene polymorphism with progression-free survival in patients with advanced urothelial carcinoma treated with the selective aurora kinase a inhibitor alisertib. Invest New Drugs. 2017;35(4):524-8. https://doi.org/ 10.1007/s10637-017-0440-5.

52. Shi $\mathrm{H}$, et al. Single nucleotide polymorphisms in the $20 \mathrm{q} 13$ amplicon genes in relation to breast cancer risk and clinical outcome. Breast Cancer Res Treat. 2011;130(3):905-16. https://doi.org/10.1007/s10549-011-1600-5.

53. Liao Y, et al. No association between FTO gene polymorphisms and central nervous system tumor susceptibility in chinese children. Pharmgenomics Pers Med. 2021;14:109-15. https://doi.org/10.2147/PGPM.S289345.

Publisher's Note Springer Nature remains neutral with regard to jurisdictional claims in published maps and institutional affiliations. 